\title{
Transesophageal echocardiography-related massive gastrointestinal bleeding during cardiac surgery
}

\author{
Hyeun Joon Bae, Kyungmi Kim, Dongho Kim, Ji-Hyun Chin, In-Cheol Choi \\ Department of Anesthesiology and Pain Medicine, Asan Medical Center, University of Ulsan College of Medicine, Seoul, Korea
}

Received October 19, 2021

Revised November 30, 2021

Accepted December 1, 2021

Corresponding author

Ji-Hyun Chin

Department of Anesthesiology and Pain Medicine, Asan Medical

Center, University of Ulsan College

of Medicine, 88 Olympic-ro 43-gil,

Songpa-gu, Seoul 05505, Korea

Tel: +82-2-3010-5632

Fax: +82-2-3010-6790

E-mail: cjh@amc.seoul.kr

ORCID:

https://orcid.org/0000-0001-9312-1685

\begin{abstract}
Transesophageal echocardiography (TEE) has become a standard intraoperative monitor during cardiac surgery. Although generally considered safe, some cases of major TEE-related complications have been reported. We present a case of a 71-year-old man with aortic aneurysm and aortic regurgitation scheduled for total arch replacement and aortic valve replacement. During the cardiopulmonary bypass period, massive gastric bleeding occurred. Emergency esophagogastroduodenoscopy (EGD) was performed during surgery and a 2-cm deep laceration was observed at the gastroesophageal junction, suggesting Mallory-Weiss syndrome caused by TEE. Since it was impossible to control massive bleeding using EGD, Sengstaken-Blakemore tube was inserted through the stomach to compress the bleeding site. Massive gastric bleeding was controlled after reversal of the effect of heparin. Since TEE might cause complications, anesthesiologists should manipulate the TEE probe gently with caution. If massive gastric bleeding is suspected during surgery, immediate EGD should be considered for diagnosis and further management.
\end{abstract}

Keywords: Transesophageal echocardiography; Cardiac surgery; Gastric bleeding; Mallory-Weiss syndrome; Sengstaken-Blakemore tube

\section{INTRODUCTION}

Transesophageal echocardiography (TEE) has become an essential intraoperative diagnostic technique for the management of patients during cardiac surgery [1,2]. TEE shows detailed real-time anatomical and physiological information of the heart, thereby providing valuable diagnostic information during cardiac surgery. Although generally considered relatively safe, some serious complications related to TEE have been reported [3,4]. When performing TEE, anesthesiologists should be aware of the potential risks of TEE and manipulate it with caution. In this report, we present a case of massive gastric bleeding due to transesophageal echocar- diography during cardiac surgery.

\section{CASE REPORT}

A 71-year-old man with an aneurysm of the descending aorta, with a maximum diameter of $72 \mathrm{~mm}$ and mild to moderate aortic regurgitation, was scheduled for total arch replacement and aortic valve replacement. His height was $164.1 \mathrm{~cm}$, and his weight was $69.7 \mathrm{~kg}$. He had a previous medical history of percutaneous coronary intervention at the left circumflex artery performed 16 years ago and atrial fibrillation. He was taking clopidogrel until 7 days and apixaban until 4 days before the current surgery. He was diag- 
nosed with liver cirrhosis Child-Pugh class A; preoperative computed tomography (CT) also revealed features of liver cirrhosis without varix or splenomegaly. Esophagogastroduodenoscopy (EGD) was not performed before the surgery. Preoperative laboratory investigations showed thrombocytopenia (platelet count of $91 \times 10^{3} / \mu \mathrm{L}$ ) and normal range of coagulation parameters (PT INR of 1.18).

In the operating room, the patient was monitored using electrocardiography, measurement of continuous arterial blood pressure over the radial and femoral arteries, non-invasive peripheral oxygen saturation measurement, bispectral index sensor, and cerebral oximetry. Anesthesia was induced and maintained with intravenous propofol, remifentanil, and rocuronium. A Multi-Lumen Access introducer and 8.5 Fr sized 4-lumen catheter were inserted at the right internal jugular vein and right subclavian vein, respectively. After lubricating the transesophageal echocardiography (TEE) probe with ultrasound gel, it was inserted through the patient's esophagus. The mid-esophageal and trans-gastric views were examined preoperatively.

Shortly after the incision, a substantial amount of crystalloid fluid and transfusion with red blood cells were required to maintain hemodynamic stability with continuous infusion of $0.2 \mathrm{mcg} / \mathrm{kg} / \mathrm{min}$ of norepinephrine. Even though four units of packed red blood cells (pRBCs) were transfused before the initiation of cardiopulmonary bypass (CPB), the hematocrit level in arterial blood gas analysis decreased from

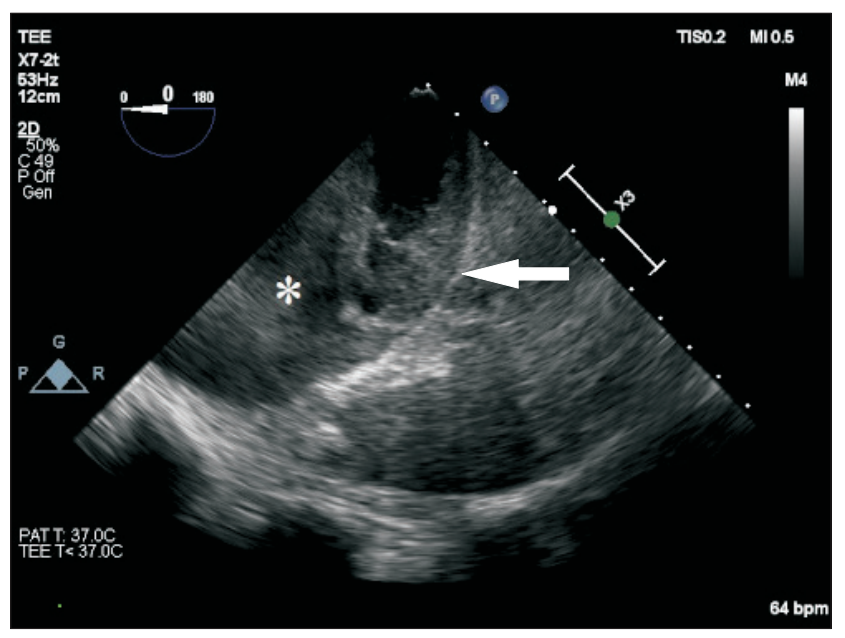

Fig. 1. Trans-gastric view of transesophageal echocardiography showing hypoechoic material suspected to be blood. The trans-gastric view of transesophageal echocardiography shows the stomach filled with hypoechoic material $\left(^{*}\right)$, suspected to be blood during cardiac surgery.
$26 \%$ to $18 \%$. Approximately $1 \mathrm{~h}$ since the initiation of $\mathrm{CPB}$, a large amount of volume loss was notified by the perfusionist and hematocrit level remained below $22 \%$ throughout this period even after the transfusion of additional four units of pRBCs. While looking for the cause of volume loss during $\mathrm{CPB}$, a large amount of blood was detected near the mouth and was continuously being shed from the mouth. We observed a distended stomach, which was full of hypoechoic material, at the trans-gastric view of TEE (Fig. 1). Gastric bleeding was strongly suspected; therefore, TEE was removed, and the nasogastric tube was inserted with negative pressure. A total of $1.5 \mathrm{~L}$ of blood was drained. Since the amount of blood draining through the nasogastric tube did not decrease, active gastric bleeding was suspected. For the diagnosis and management of the bleeding, emergency EGD was performed by gastroenterologists in the operating room. However, visualizing through the scope was difficult due to the massive bleeding, and the focus of bleeding could not be located. During EGD, a hypothermic circulatory arrest was performed as a surgical procedure. Shortly thereafter, the gastric bleeding reduced, and a $2 \mathrm{~cm}$ sized deep laceration was found near the gastroesophageal (GE) junction (Fig. 2). The laceration was diagnosed as a procedure-related injury at the mucosa, not involving the esophageal varix. Controlling the bleeding with endoscopic intervention was challenging, owing to the difficulty in locating the focus

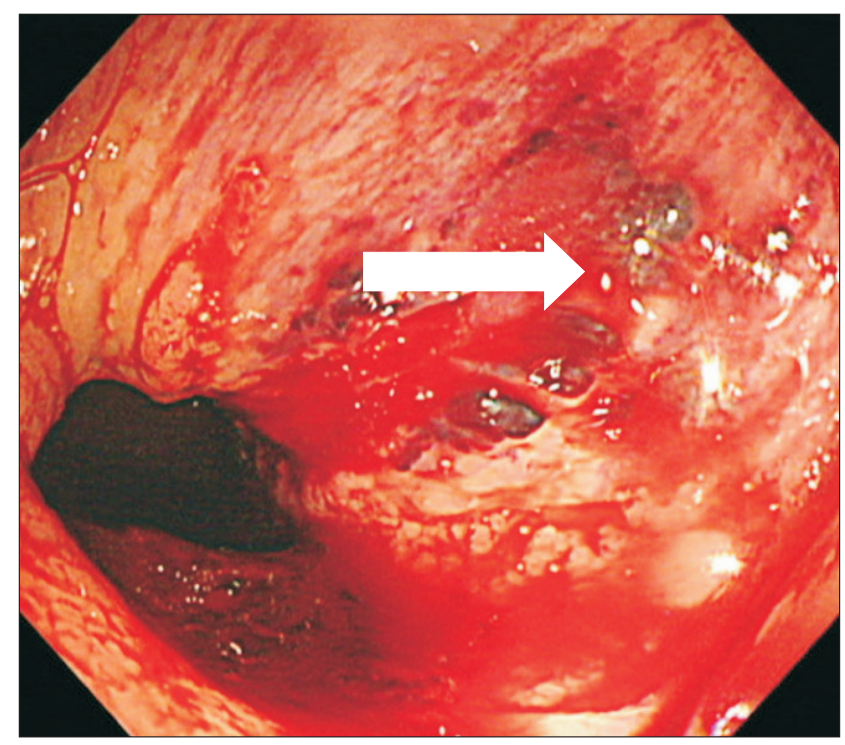

Fig. 2. Esophagogastroduodenoscopy showing Mallory-Weiss syndrome. Esophagogastroduodenoscopy shows a 2-cm sized deep laceration (arrow) with active bleeding during cardiac surgery under cardiopulmonary bypass. 
of the bleeding after resuming circulation under $\mathrm{CPB}$. The Sengstaken-Blakemore tube (SB tube) was decided to be inserted through the esophagus. However, traction could not be applied to the SB tube due to the limited space in the operating room. Gastric bleeding continued even after applying the SB tube during $\mathrm{CPB}$. After completion of the main procedure, the effects of heparin were reversed by administering intravenous protamine. Simultaneously, coagulopathy was corrected with fresh frozen plasma, cryoprecipitate, platelet concentrate, and tranexamic acid. The draining of blood through the SB tube started to decrease considerably. The total duration of anesthesia was $12 \mathrm{~h}$ and $50 \mathrm{~min}$, while the total duration of $\mathrm{CPB}$ was $5 \mathrm{~h}$ and $43 \mathrm{~min}$.

After the surgery, the patient was transferred to the cardiac surgery intensive care unit (ICU). In the ICU, traction was applied to the SB tube to control continuous bleeding, after confirming its location on the chest radiograph. EGD was performed the following day after removing the SB tube, but the site of laceration was not visible due to hematoma in the esophagus with no signs of active gastric bleeding. In addition, multiple venous dilations were detected from the low to mid esophagus without a red colored sign on EGD. On postoperative day (POD) 6, EGD was performed again, and a 2-cm-sized healed linear laceration was found at the cardiac-pyloric wall (Fig. 3). No fatal gastrointestinal complications needing further management, including active

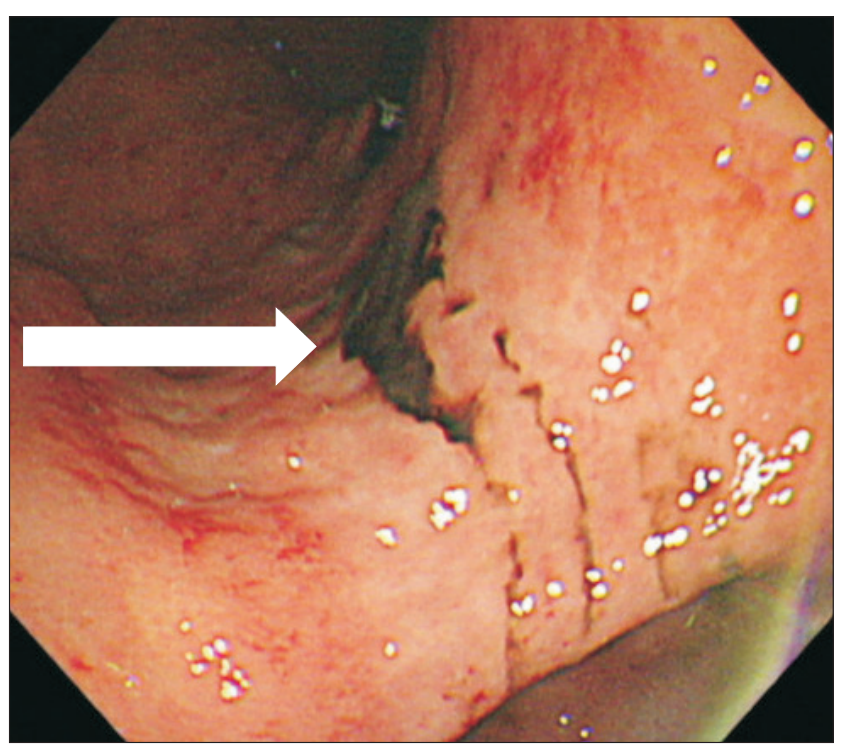

Fig. 3. Esophagogastroduodenoscopy showing healed ulceration. Esophagogastroduodenoscopy shows a linear healed ulceration (arrow) at postoperative day 6. gastric bleeding or esophageal perforation, were found. The patient was extubated on POD 10 and transferred to the general ward on POD 16.

\section{DISCUSSION}

TEE is an effective diagnostic tool for guiding the surgical procedure and detecting unexpected problems during cardiac surgery. The recent American Society of Anesthesiologists guidelines recommend that TEE should be used in all patients going through thoracic aorta surgery without contraindications [1]. During the surgery of the thoracic aorta, TEE is useful in diagnosing aneurysms, confirming the correct placement of the cannula, and closely monitoring the cardiac function [2].

TEE is largely considered a safe diagnostic modality with a low complication profile. However, some cases of major complications have been reported. Major gastric bleeding, caused by direct contact of the TEE with the mucosal wall or esophageal varices has been reported in $0.02-1.0 \%$ of the cases [3]. In this case, the linear laceration at the GE junction on EGD was diagnosed as TEE-related injury or the Mallory-Weiss syndrome caused by TEE. Mallory-Weiss syndrome is characterized by longitudinal, non-perforating mucosal lacerations in the GE junction, resulting in gastric bleeding. We believe that the laceration in our case might have occurred while obtaining the deep trans-gastric view with the TEE. Extreme anteflexion of the TEE probe at the GE junction might exert some pressure on the mucosal wall, leading to Mallory-Weiss syndrome. Despite lubricating the TEE probe with gel and managing the TEE probe with caution, we reason that the TEE probe should be manipulated more carefully, including avoiding extreme anteflexion. Manipulating the probe with adequate caution can prevent injuries related to TEE [3]. Before the insertion, the TEE probe should be checked for any damages, and unlocking of the probe should be reconfirmed [5]. Since manipulation of the probe when locked in extreme anteflexion can cause significant injury to the esophagus and stomach, probe insertion and manipulation in the locked position must be avoided. In addition, applying adequate lubrication to the probe decreases the friction between the mucosa and the probe, thereby preventing injury to the mucosal wall.

Considering that heparin is injected during the $\mathrm{CPB}$ peri- 
od and coagulopathy occurs after weaning from $\mathrm{CPB}$, cardiac surgery increases the risk of TEE-related bleeding [3]. The use of heparin during СРB itself might be the cause of gastric bleeding regardless of the use of TEE during cardiac surgery. One study reported that gastric bleeding occurred in $0.26 \%$ of patients after cardiac surgery without the use of TEE [6].

In our case, there was no delay in the surgery, since efforts to stop the gastric bleeding were made simultaneously with the surgical procedure. However, coagulopathy aggravated by massive gastric bleeding might cause difficulty in hemostasis after weaning from $\mathrm{CPB}$, leading to an increased total operating time. Moreover, massive gastric bleeding and substantially aggravated coagulopathy could have led to increased amount of pRBC transfusion. A total of 35 units of pRBCs was transfused in our case, which was significantly greater compared to a previous single center study where a median eight units of pRBCs were transfused during total arch replacement surgery [7]. Increased amount of transfusion during cardiac surgery was reported to be associated with higher morbidity and mortality rate [8-10]. Therefore, we could not exclude the possibility that massive gastric bleeding during the surgery negatively affected our patient's postoperative outcome.

In our case, preoperative CT showed liver cirrhosis, but not esophageal varices, and the existence of esophageal varices was not confirmed with preoperative EGD. Given that our case was diagnosed as a TEE related injury on EGD, esophageal variceal bleeding was not applicable. However, preoperative existence of esophageal varices could not be completely excluded, considering that postoperative EGD featured esophageal varices. Patients with esophageal varices need to be managed with caution because esophageal varix is an important factor in causing TEE-related gastric bleeding. Blind manipulation of the probe in the esophagus might increase the risk of variceal bleeding, although patients with liver cirrhosis and esophageal varices have been reported to have a low incidence of variceal bleeding after TEE examination in cases of small varices [11]. A previous study reported a $2.5 \%$ rate of bleeding in patients with high model for end-stage liver disease scores, low platelet counts, and high INR [12]. The American Society of Echocardiography and the Society of Cardiovascular Anesthesiologists consider the presence of esophageal varices as a relative contraindication for TEE and recommend obtaining approval from a gastroenterologist before performing TEE. The American College of Gastroenterology also suggests endoscopic surveillance for gastroesophageal varices in patients with liver cirrhosis before performing TEE.

In conclusion, we experienced a case of massive gastric bleeding due to laceration at the GE junction caused by TEE. Although TEE is a useful and safe diagnostic tool, anesthesiologists should be aware of the potential risks of complications, including major bleeding; gentle manipulation with caution might reduce the damage caused by TEE.

\section{CONFLICT OF INTEREST}

No potential conflict of interest relevant to this article was reported.

\section{REFERENCES}

1. American Society of Anesthesiologists and Society of Cardiovascular Anesthesiologists Task Force on Transesophageal Echocardiography. Practice guidelines for perioperative transesophageal echocardiography. An updated report by the American Society of Anesthesiologists and the Society of Cardiovascular Anesthesiologists Task Force on Transesophageal Echocardiography. Anesthesiology 2010;112:1084-96.

2. Nowak-Machen M. The role of transesophageal echocardiography in aortic surgery. Best Pract Res Clin Anaesthesiol 2016;30:317-29.

3. Hilberath JN, Oakes DA, Shernan SK, Bulwer BE, D'Ambra MN, Eltzschig HK. Safety of transesophageal echocardiography. J Am Soc Echocardiogr 2010;23:1115-27; quiz 1220-1.

4. Lee JY, Jeong DM, Lee SH, Lee SM. Transesophageal echocardiography (TEE)-induced Mallory-Weiss laceration in a patient who underwent aortic and mitral valve replacement -a case report-. Korean J Anesthesiol 2010;59 Suppl:S103-6.

5. Hahn RT, Abraham T, Adams MS, Bruce CJ, Glas KE, Lang RM, et al. Guidelines for performing a comprehensive transesophageal echocardiographic examination: recommendations from the American Society of Echocardiography and the Society of Cardiovascular Anesthesiologists. J Am Soc Echocardiogr 2013;26:921-64.

6. Egleston CV, Wood AE, Gorey TF, McGovern EM. Gastrointestinal complications after cardiac surgery. Ann R Coll Surg Engl 1993;75:52-6.

7. Li CN, Ge YP, Liu H, Zhang CH, Zhong YL, Chen SW, et al. Blood transfusion and acute kidney injury after total aortic arch replacement for acute stanford type a aortic dissection. 
Heart Lung Circ 2022;31:136-43.

8. Velasquez CA, Singh M, Bin Mahmood SU, Brownstein AJ, Zafar MA, Saeyeldin A, et al. The effect of blood transfusion on outcomes in aortic surgery. Int J Angiol 2017;26:135-42.

9. Ming Y, Liu J, Zhang F, Chen C, Zhou L, Du L, et al. Transfusion of red blood cells, fresh frozen plasma, or platelets is associated with mortality and infection after cardiac surgery in a dose-dependent manner. Anesth Analg 2020;130:48897.

10. Vlot EA, Verwijmeren L, van de Garde EMW, Kloppenburg GTL, van Dongen EPA, Noordzij PG. Intra-operative red blood cell transfusion and mortality after cardiac surgery.
BMC Anesthesiol 2019;19:65.

11. Nigatu A, Yap JE, Lee Chuy K, Go B, Doukky R. Bleeding risk of transesophageal echocardiography in patients with esophageal varices. J Am Soc Echocardiogr 2019;32:674-6. e2.

12. Myo Bui CC, Worapot A, Xia W, Delgado L, Steadman RH, Busuttil RW, et al. Gastroesophageal and hemorrhagic complications associated with intraoperative transesophageal echocardiography in patients with model for end-stage liver disease score 25 or higher. J Cardiothorac Vasc Anesth 2015;29:594-7. 